\title{
Concurrent Use of Teneligliptin and Canagliflozin Improves Glycemic Control with Beneficial Effects on Plasma Glucagon and Glucagon-Like Peptide-1: A Single-Arm Study
}

\author{
Tomoho Noda $\cdot$ Emi Ebihara $\cdot$ Hiroaki Ueno (D) - Keisuke Sadohara \\ Yuri Tanaka · Yuuma Nagatomo • Yousuke Murakami • \\ Shinichi Yonamine - Wakaba Tsuchimochi · Hideyuki Sakoda • \\ Hideki Yamaguchi · Masamitsu Nakazato
}

Received: May 21, 2019 / Published online: July 12, 2019

(C) The Author(s) 2019

\section{ABSTRACT}

Introduction: We investigated the mechanisms of the glucose-lowering effects of teneligliptin and canagliflozin, a sodium-glucose cotransporter-2 (SGLT2) inhibitor, by monitoring several gastrointestinal peptides using the most appropriate measuring methods during multiple meal tolerance tests (MTTs) and flash glucose monitoring.

Enhanced Digital Features To view enhanced digital features for this article go to https://doi.org/10.6084/ m9.figshare. 8483387 .

Electronic supplementary material The online version of this article (https://doi.org/10.1007/s13300019-0666-7) contains supplementary material, which is available to authorized users.

Tomoho Noda and Emi Ebihara are equally contributed first author.

T. Noda $\cdot$ E. Ebihara $\cdot$ H. Ueno $(\bowtie) \cdot$ K. Sadohara

Y. Tanaka - Y. Nagatomo - Y. Murakami .

S. Yonamine · W. Tsuchimochi · H. Sakoda .

H. Yamaguchi · M. Nakazato $(\bowtie)$

Division of Neurology, Respirology, Endocrinology and Metabolism, Department of Internal Medicine, Faculty of Medicine, University of Miyazaki,

Miyazaki 889-1692, Japan

e-mail: intron@med.miyazaki-u.ac.jp

M. Nakazato

e-mail: nakazato@med.miyazaki-u.ac.jp
Methods: Twelve Japanese patients with type 2 diabetes were enrolled in the 14-day study. Subjects were treated with teneligliptin $20 \mathrm{mg}$ /day from day 4 , followed by a combination tablet of teneligliptin $20 \mathrm{mg}$ and canagliflozin $100 \mathrm{mg}$ (T/C) per day from day 11. MTTs were conducted on days 3 (premedication; Pre), 10 (teneligliptin; T) and 13 (T/C) to evaluate plasma glucose, C-peptide, glucagon, active glucagon-like peptide-1 (GLP-1), active gastric inhibitory polypeptide (GIP), ghrelin and desacyl ghrelin.

Results: Plasma glucose was significantly decreased with the progress of treatment intervention, and C-peptide was significantly decreased in $\mathrm{T} / \mathrm{C}$ compared to the others. Plasma postprandial glucagon was increased for $90 \mathrm{~min}$ from fasting in Pre, but only for $30 \mathrm{~min}$ in $\mathrm{T}$ and $\mathrm{T} / \mathrm{C}$. Plasma postprandial active GLP-1 was significantly increased in $\mathrm{T}$ compared to Pre, and that of $\mathrm{T} / \mathrm{C}$ was significantly higher than T. Plasma postprandial active GIP was increased in $\mathrm{T}$ and $\mathrm{T} / \mathrm{C}$ compared to Pre. Plasma ghrelin and des-acyl ghrelin levels did not change during the treatment.

Conclusion: Teneligliptin increased incretin hormones and suppressed postprandial glucagon secretion as expected. Concurrent use of canagliflozin and teneligliptin improved glycemic control without increasing postprandial glucagon secretion, and increased postprandial GLP-1 secretion and decreased the required 
amount of postprandial insulin secretion. The underlying mechanisms may involve canagliflozin's inhibitory activity against not only SGLT2 but also SGLT1.

Trial Registration: UMIN identifier, UMIN000030043.

Funding: Mitsubishi Tanabe Pharma Corporation and a Grant for Clinical Research from Miyazaki University Hospital.

Keywords: Canagliflozin; Glucagon; Glucagonlike peptide-1; Insulin; Teneligliptin

\section{INTRODUCTION}

Standard treatment for type 2 diabetes mellitus consists of dietary therapy, exercise therapy and pharmacotherapy. In recent years, new drugs with different mechanisms of action have been developed for the treatment of type 2 diabetes. Dipeptidyl peptidase-4 (DPP-4) inhibitors promote glucose-dependent insulin secretion and suppress glucagon secretion by inhibiting the degradation of glucagon-like peptide-1 (GLP-1) and glucosedependent insulinotropic polypeptide (GIP) by binding to DPP-4, thereby exerting a glucose-lowering effect. Since DPP-4 inhibitors bring a low risk of hypoglycemia, approximately $70 \%$ of Japanese type 2 diabetes patients were prescribed the drug in 2014 following its launch in 2009 [1].

Sodium-glucose cotransporter-2 (SGLT2) inhibitors lower blood glucose levels by inhibiting SGLT2 in the proximal tubule and increasing glucose excretion in the urine [2]. The CANVAS Program, a large clinical trial assessing the cardiovascular safety of the SGLT2 inhibitor canagliflozin, showed a significant reduction of cardiovascular events in the group administered canagliflozin compared to the placebo group [3].

In clinical practice, SGLT2 inhibitors are often used as add-on therapy when the effect of DPP-4 inhibitors is insufficient; however, the changes in feeding-regulatory peptides, including GLP-1 and GIP, and glucose-lipid metabolism associated with such add-on therapy have not been fully elucidated. We therefore conducted this study to investigate the mechanisms of the hypoglycemic action of combination therapy with DPP-4 and SGLT2 inhibitors.

\section{METHODS}

\section{Study Participants}

The subjects were type 2 diabetes mellitus patients, aged 20-75 years, who were seen at the Department of Endocrinology, Metabolism and Diabetes of our hospital between June and December 2018. The inclusion criteria were: patients who were not taking oral hypoglycemic drugs or metformin alone, and with a HbA1c level of $7-11 \%$. The exclusion criteria were: patients not seen regularly by medical staff, presence of proliferative diabetic retinopathy, dialysis treatment, history of hypersensitivity to teneligliptin or canagliflozin, severe ketosis, diabetic coma or precoma, severe infection, in the perioperative period, severe trauma, insulin or GLP-1 receptor agonist use, and geriatric syndrome. Thirteen patients participated in this study after providing informed consent. One patient withdrew from the study because of lacunar infarction that occurred on the 7 th day after starting teneligliptin, although the physical symptoms associated with lacunar infarction essentially disappeared by the time of discharge. The remaining 12 patients completed the study protocol and were included in the analysis. Table 1 shows the characteristics of the 12 patients. We defined hypertension, dyslipidemia, albuminuria and diabetic neuropathy as taking antihypertensive agent, taking antidyslipidemic agent, albuminuria 30-299 mg/day and distal symmetric polyneuropathy with vibration sense reduction $(<10 \mathrm{~s})$ at medial malleolus, respectively.

\section{Study Protocol and Ethical Statement}

All data in this study were collected at our hospital. This study was a single arm open-label trial. This study was approved by the Ethics Review Committee of the University of Miyazaki and is registered in the University Hospital Medical Information Network Clinical Trials Registry as UMIN000030043. All procedures were in accordance with the ethical standards of the responsible committee on human experimentation (institutional and national) and the 
Table 1 Baseline characteristics of subjects

\begin{tabular}{ll}
\hline Characteristics & Value \\
\hline Age (years) & $61.3 \pm 2.5$ \\
Sex (men/women) & $3 / 9$ \\
Body mass index $\left(\mathrm{kg} / \mathrm{m}^{2}\right)$ & $27.8 \pm 5.5$ \\
HbA1c (\%) & $7.7 \pm 0.7$ \\
Fasting blood glucose $(\mathrm{mg} / \mathrm{dL})$ & $132.4 \pm 27.4$ \\
Duration of T2DM (year) & $2.4 \pm 3.4$ \\
Hypertension/dyslipidemia & $3 / 7$ \\
Treatment & \\
Diet only & 8 \\
Metformin $(250-1000 \mathrm{mg} /$ day) & 4 \\
Diabetic complication & \\
Retinopathy (none/simple) & $10 / 2$ \\
Nephropathy (none/albuminuria) & $9 / 3$ \\
Neuropathy (-/+) & $11 / 1$ \\
\hline
\end{tabular}

Values are mean $\pm \mathrm{SD}$

T2DM type 2 diabetes mellitus

Helsinki Declaration of 1964, as revised in 2013. Patient registration was made by physicians participating in this study. An intervention study was performed during 14 days of hospitalization after obtaining written informed consent to participate from all patients. Patients received a diet of $25-30 \mathrm{kcal} / \mathrm{kg}$ ideal body weight with $6 \mathrm{~g}$ sodium chloride per day during hospitalization. Teneligliptin $20 \mathrm{mg} /$ day was started on day 4 of hospitalization and changed to a combination tablet of teneligliptin $20 \mathrm{mg}$ and canagliflozin $100 \mathrm{mg}$ per day on day 11 . Meal tolerance tests (MTT) were performed on day 3 of hospitalization before starting teneligliptin (Pre), the 7th day after starting teneligliptin (T) and the 3rd day after changing to the combination tablet of teneligliptin and canagliflozin (T/C) (Fig. S1). The amount of water intake was measured and urine collection was performed for 3 days in each period, and urine volume, urinary C-peptide (CPR), urine glucose, urine protein and urine albumin were measured. During hospitalization, 24-h continuous blood glucose levels were measured using the FreeStyle Libre ${ }^{\circledR}$ flash glucose monitoring (FGM) system (Fig. S1). Body weight, blood pressure, pulse rate, lipids, uric acid, acetoacetic acid, 3-hydroxybutyric acid, total ketone bodies, and liver and renal function were measured in the morning while fasting on the day of the 3 MTTs.

\section{Study Endpoints}

The primary endpoints were changes in blood glucose levels at all time-points of the 3 MTTs and the area under the curve (AUC) of glucose levels and changes in blood glucose control in the FGM data of Pre, T and T/C [mean blood glucose level, standard deviation (SD), mean amplitude of glucose excursion (MAGE), time above $180 \mathrm{mg} / \mathrm{dL}$ and time below $70 \mathrm{mg} / \mathrm{dL}$ ]. The secondary endpoints were changes in the following parameters: insulin, CPR, active GLP1 , active GIP, glucagon, ghrelin and des-acyl ghrelin levels at all time-points of the 3 MTTs and each AUC, body weight, blood pressure, pulse rate, amount of water intake, urine volume, urinary $\mathrm{CPR}$, urine glucose, urine protein, urine albumin, lipids, renal function, uric acid, liver function, high sensitivity- $C$ reactive protein (hs-CRP), acetoacetic acid, 3-hydroxybutyric acid and total ketone bodies.

\section{Blood Sample Processing}

Blood glucose and CPR levels were measured with a hexokinase assay and chemiluminescent enzyme immunoassay (SRL Inc, Tokyo, Japan), respectively. For measurements of blood active GLP-1, active GIP and glucagon, blood was collected using P-800 blood collection tubes (Japan Becton, Dickinson and Company, Tokyo, Japan), and the tubes were centrifuged at $1468 \mathrm{~g}$ for $15 \mathrm{~min}$ at $4{ }^{\circ} \mathrm{C}$ to isolate plasma. The samples for glucagon measurements were immediately placed in a freezer at $-30^{\circ} \mathrm{C}$, and glucagon levels were measured by a sandwich enzyme-linked immunosorbent assay (ELISA) using $\mathrm{N}$ - and C-terminal specific antibodies (Mercodia AB, Uppsala, Sweden). The samples 
for active GLP-1 and active GIP measurements were extracted using OASIS HLB (Nihon Waters, Tokyo, Japan). Active GLP-1 and active GIP levels were measured with the active GLP-1 ELISA kit (Merck, Darmstadt, Germany) and the active GIP ELISA kit (Yanaihara Inc, Shizuoka, Japan), respectively. To measure ghrelin and des-acyl ghrelin levels, blood was collected directly into tubes containing aprotinin; the tubes were immediately centrifuged for plasma isolation at $4{ }^{\circ} \mathrm{C}$. The isolated plasma was treated with one-tenth of its volume of $1 \mathrm{~N} \mathrm{HCl}$ and the tubes were rocked gently. These samples were then used for plasma ghrelin and des-acyl ghrelin measurements using an automated enzyme immunoassay (AIA-600II, Tosoh Corp, Tokyo, Japan) as described elsewhere [4].

The SUIT, an index of insulin secretion ability in response to postprandial glucose elevation, was calculated using the formula $[1500 \times$ fasting $\mathrm{CPR} /($ fasting plasma glucose - 61.7)] [5]. The CPR-IR, an index of insulin resistance, was calculated using the formula [20/(fasting CPR $\times$ fasting plasma glucose)] [6]. The HOMA2-\%B, an index of insulin secretion ability, was calculated using software [7]. AUC was calculated according to the trapezoidal method.

\section{Meal Tolerance Test}

MTTs $(592 \mathrm{kcal}$, carbohydrate $75 \mathrm{~g}$, protein $8.0 \mathrm{~g}$, fat $28.5 \mathrm{~g}$, Saraya Corp, Osaka, Japan) were conducted three times: Pre (day 3), T (day 10) and T/C (day 13) (Fig. S1). Blood samples were taken at 0 (baseline), 30, 60, 90, 120 and 180 min postprandial; blood glucose, insulin, CPR, glucagon, active GLP-1, active GIP, ghrelin and des-acyl ghrelin levels were measured at all blood sampling times. Hemolysis occurred in a few samples, precluding the measurement of insulin in all samples.

\section{Statistical Analysis}

The results are expressed as mean $\pm \mathrm{SD}$. Comparison of the 3 MTTs in total was performed using a repeated-measures ANOVA. The changes in each measurement item such as AUC blood glucose level in the MTT, changes in blood glucose control in the FGM data (mean blood glucose level, SD, MAGE, time above $180 \mathrm{mg} / \mathrm{dL}$ and time below $70 \mathrm{mg} / \mathrm{dL}$ ) and each test parameter of the 3 MTTs were analyzed by a repeated-measures ANOVA and the Holm-Sidak post hoc test using the statistical software JMP12 and Prism 7.

\section{RESULTS}

The blood glucose levels of MTTs showed significant differences between the three periods (Pre, $\mathrm{T}$ and $\mathrm{T} / \mathrm{C}$ ). Analysis by time showed that the values were significantly lower in $\mathrm{T}$ and $\mathrm{T} / \mathrm{C}$ at all time points between 0 and $180 \mathrm{~min}$ compared with Pre. Blood glucose levels in T/C between 30 and $180 \mathrm{~min}$ were significantly lower than those in T. The AUC glucose was also significantly lower in $\mathrm{T}$ and $\mathrm{T} / \mathrm{C}$ compared with Pre, and that of $\mathrm{T} / \mathrm{C}$ was significantly lower than that of $\mathrm{T}$ (Fig. 1a, b). The mean blood glucose level, SD and MAGE obtained from the FGM data were significantly decreased in the order of Pre, $\mathrm{T}$ and $\mathrm{T} / \mathrm{C}$. The time above $180 \mathrm{mg} / \mathrm{dL}$ was significantly lower in $\mathrm{T}$ and $\mathrm{T} / \mathrm{C}$ compared with Pre, whereas the time below $70 \mathrm{mg} / \mathrm{dL}$ did not significantly differ between the three periods (Table 2).

The CPR of MTTs was significantly lower in $\mathrm{T} / \mathrm{C}$ compared with Pre and $\mathrm{T}$ (between 60 and $180 \mathrm{~min})$, and that of $\mathrm{T}$ at $90 \mathrm{~min}$ was significantly lower than Pre. The AUC CPR was significantly lower in $\mathrm{T} / \mathrm{C}$ compared with Pre and $\mathrm{T}$ (Fig. 1c, d). The $\Delta$ CPR-30 min/ $\Delta$ glucose-30 min, an index of early phase insulin secretion, was significantly higher in $\mathrm{T}(3.50 \pm 2.86, P=0.024$ vs Pre) and T/C $(4.30 \pm 3.15, P=0.012$ vs Pre $)$ than in Pre $(1.64 \pm 1.30)$. The SUIT, an index of insulin secretion ability in response to postprandial glucose elevation, was significantly improved in $\mathrm{T}$ and $\mathrm{T} / \mathrm{C}$ compared with Pre. The CPR-IR, an index of insulin resistance, was significantly improved in T/C compared with Pre and T. The HOMA2- $\% \mathrm{~B}$, an index of insulin secretion ability, was significantly increased in the order of Pre, T and T/C (Table 2). The total amount of insulin secretion in response to glucose (AUC-CPR ${ }_{0-180} /$ AUC-glucose $\left._{0-180}\right)$ was 
A

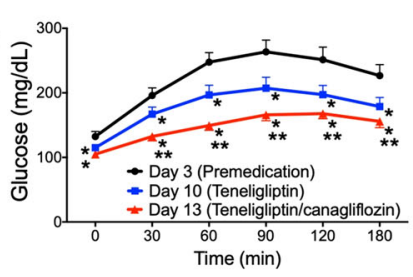

C

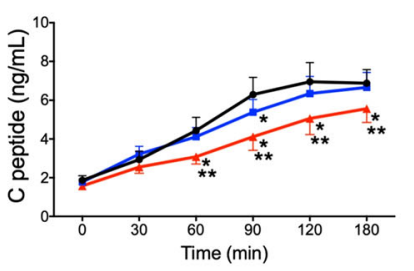

E

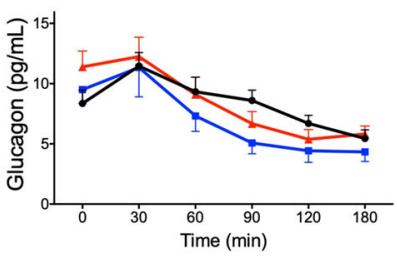

G

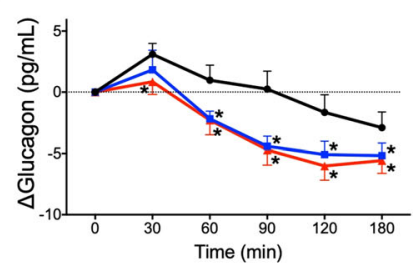

I

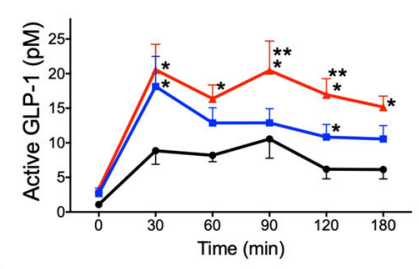

K

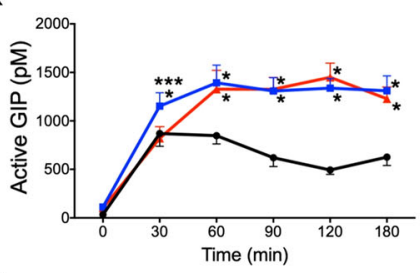

M

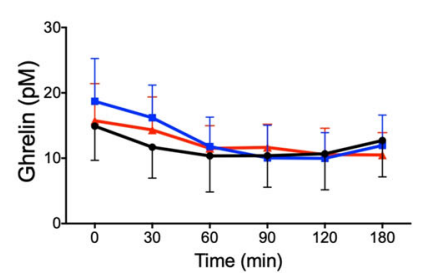

B

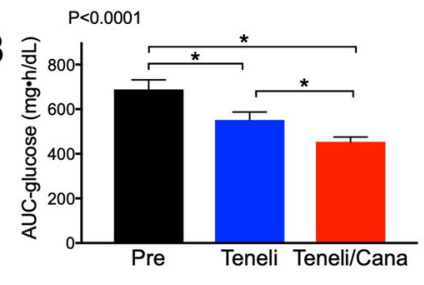

D $P=0.0004$

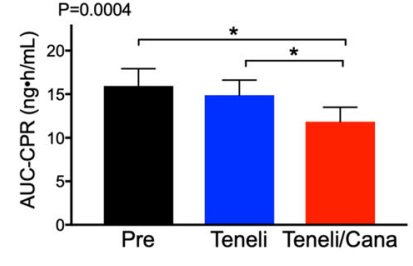

$\mathbf{F}$

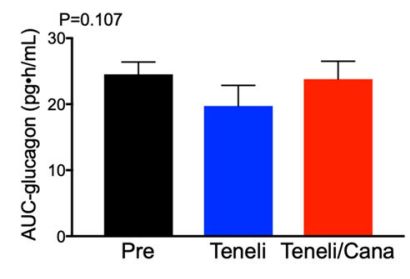

H $\quad P=0.0012$

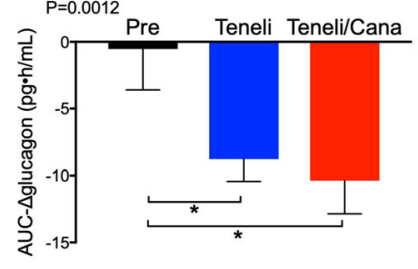

J

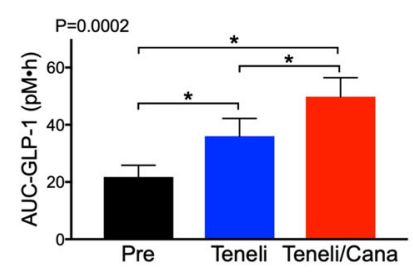

$\mathbf{L}$

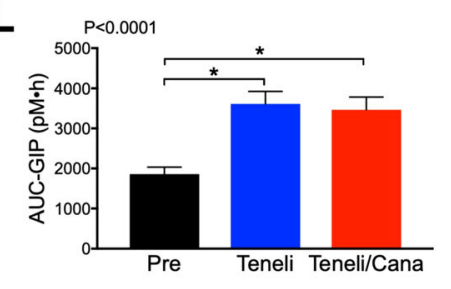

$\mathbf{N}$

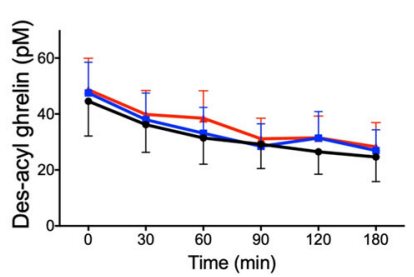


4Fig. 1 Effects of teneligliptin and canagliflozin on glucose, insulin, glucagon and gastrointestinal peptide responses to meal tolerance tests. The changes in a plasma glucose, b glucose $\mathrm{AUC}_{0-180}$, c serum C-peptide, d C-peptide $\mathrm{AUC}_{0-180}$, e plasma glucagon, f glucagon $\mathrm{AUC}_{0-180}$, g plasma $\Delta$ glucagon, h $\Delta$ glucagon $\mathrm{AUC}_{0-180}$, i plasma active GLP-1, $\mathbf{j}$ active GLP-1 AUC $_{0-180}$, $\mathbf{k}$ plasma active GIP, 1 active GIP $\mathrm{AUC}_{0-180}$, $\mathbf{m}$ plasma ghrelin and n plasma des-acyl ghrelin. Black, blue and red lines indicate day 3 (premedication; Pre), day 10 (teneligliptin; Teneli) and day 13 (combination tablet of teneligliptin and canagliflozin; Teneli/Cana), respectively. $P$ values in $\mathbf{b}, \mathbf{d}, \mathbf{f}$, $\mathbf{h}, \mathbf{j}$ and $\mathbf{l}$ were analyzed by a repeated-measures ANOVA. Data are mean \pm SE. ${ }^{*} P<0.05$ vs day $3,{ }^{* *} P<0.05$ vs day $10,{ }^{* * *} P<0.05$ vs day 13

significantly higher in $\mathrm{T} \quad(28.0 \pm 11.7$, $P=0.039)$ but not in $\mathrm{T} / \mathrm{C} \quad(26.3 \pm 11.9$, $P=0.27)$ compared to Pre $(23.7 \pm 9.6)$.

Before starting the medication, plasma glucagon remained at a higher level for $90 \mathrm{~min}$ postprandial compared with preprandial values and subsequently decreased. In $\mathrm{T}$ and $\mathrm{T} / \mathrm{C}$, glucagon increased at only $30 \mathrm{~min}$ postprandial and subsequently decreased (Fig. 1g). Plasma glucagon levels in MTTs and AUC glucagon showed no significant differences between the three periods. However, the $\Delta$ glucagon (compared to preprandial values of MTT) was significantly lower in $\mathrm{T}$ (between 60 and $180 \mathrm{~min}$ ) and T/C (between 30 and $180 \mathrm{~min}$ ) compared with Pre. The AUC $\Delta$ glucagon was significantly lower in $\mathrm{T}$ and $\mathrm{T} / \mathrm{C}$ than in Pre (Fig. 1e-h). The active GLP-1 level in $\mathrm{T}$ was significantly higher at 30 and 120 min postprandial compared with Pre, and that in T/C was significantly higher at 30-180 min postprandial compared with Pre. The active GLP-1 level in T/C was significantly higher at 90-120 min postprandial compared with T. The AUC active GLP-1 was significantly higher in $\mathrm{T}$ and $\mathrm{T} / \mathrm{C}$ compared with Pre, and that of $\mathrm{T} / \mathrm{C}$ was significantly higher than that of $\mathrm{T}$ (Fig. 1i, j). The active GIP level was significantly higher in $\mathrm{T}$ and T/C 60 min postprandial compared with Pre, and that of $\mathrm{T}$ at $30 \mathrm{~min}$ was significantly higher than that of Pre and T/C. The AUC active GIP was significantly higher in $\mathrm{T}$ and $\mathrm{T} / \mathrm{C}$ compared with Pre (Fig. 1k, l). No significant changes were observed in ghrelin or des-acyl ghrelin (Fig. 1m, n).

Table 3 shows the changes in body weight, blood pressure, pulse rate, urine volume, and amount of water intake. Body weight and body mass index were significantly decreased in the order of Pre, $\mathrm{T}$ and T/C. Both systolic and

Table 2 Indices of blood glucose fluctuations measured by flash glucose monitoring, insulin secretion and insulin resistance

\begin{tabular}{|c|c|c|c|c|}
\hline & Pre & Teneligliptin & Teneli/Cana & $P$ value \\
\hline 24-h mean glucose levels $(\mathrm{mg} / \mathrm{dL})$ & $155.0 \pm 38.9$ & $128.4 \pm 21.8^{*}$ & $113.2 \pm 13.8^{*} \dagger$ & 0.0009 \\
\hline $\mathrm{SD}$ of $24-\mathrm{h}$ glucose levels $(\mathrm{mg} / \mathrm{dL})$ & $45.9 \pm 15.7$ & $32.9 \pm 11.4^{*}$ & $26.5 \pm 9.1^{* \dagger}$ & 0.0005 \\
\hline $\operatorname{MAGE}(\mathrm{mg} / \mathrm{dL})$ & $124.1 \pm 45.6$ & $93.7 \pm 43.9^{*}$ & $65.6 \pm 29.7^{*} \dagger$ & 0.0009 \\
\hline $\mathrm{CV}(\%)$ & $29.5 \pm 7.1$ & $25.0 \pm 5.0^{*}$ & $23.1 \pm 6.3^{*}$ & 0.0029 \\
\hline \multicolumn{5}{|l|}{ Proportion (\%) of time of } \\
\hline Hyperglycemia (> $180 \mathrm{mg} / \mathrm{dL})$ & $27.2 \pm 26.2$ & $8.7 \pm 12.4^{*}$ & $3.4 \pm 6.4^{*}$ & 0.010 \\
\hline Hypoglycemia $(<70$ mg/dL) & $0.35 \pm 0.68$ & $0.09 \pm 0.30$ & $1.40 \pm 4.86$ & 0.42 \\
\hline SUIT & $41.8 \pm 17.4$ & $53.8 \pm 22.6^{*}$ & $59.5 \pm 26.6^{*}$ & 0.0005 \\
\hline CPR-IR & $5.5 \pm 2.8$ & $6.0 \pm 2.1$ & $7.4 \pm 2.8^{* \dagger}$ & 0.012 \\
\hline HOMA2-\%B & $134.4 \pm 44.7$ & $164.2 \pm 53.9^{*}$ & $176.1 \pm 56.8^{*} \dagger$ & $<0.0001$ \\
\hline
\end{tabular}

Values are mean $\pm S D$

Pre premedication, Teneli/Cana combination tablet of teneligliptin and canagliflozin, MAGE mean amplitude of glucose excursion, SUIT secretory units of islets in transplantation

${ }^{*} P<0.05$ vs Pre, ${ }^{\dagger} P<0.05$ vs teneligliptin 
Table 3 Changes in the parameters

\begin{tabular}{lcccc}
\hline & Pre & Teneligliptin & Teneli/Cana & P value \\
\hline Body weight $(\mathrm{kg})$ & $65.2 \pm 12.7$ & $64.5 \pm 12.6^{*}$ & $63.8 \pm 12.5^{*} \dagger$ & $<0.0001$ \\
BMI $\left(\mathrm{kg} / \mathrm{m}^{2}\right)$ & $27.2 \pm 5.7$ & $26.9 \pm 5.6^{*}$ & $26.6 \pm 5.6^{*} \dagger$ & $<0.0001$ \\
SBP $(\mathrm{mmHg})$ & $132.0 \pm 10.1$ & $120.2 \pm 109$ & $117.9 \pm 10.1^{*}$ & 0.0003 \\
DBP $(\mathrm{mmHg})$ & $76.4 \pm 12.2$ & $70.9 \pm 10.2$ & $70.3 \pm 8.4^{*}$ & 0.042 \\
Pulse rate $(/ \mathrm{min})$ & $72.1 \pm 9.6$ & $71.2 \pm 8.8$ & $72.8 \pm 8.0$ & 0.61 \\
Urine volume $(\mathrm{mL} /$ day $)$ & $1579 \pm 673$ & $1785 \pm 482$ & $1765 \pm 399$ & 0.19 \\
Water intake $(\mathrm{mL} /$ day $)$ & $1462 \pm 615$ & $1624 \pm 487$ & $1708 \pm 362$ & 0.13 \\
\hline
\end{tabular}

Values are mean $\pm S D$

Pre premedication, Teneli/Cana combination tablet of teneligliptin and canagliflozin

${ }^{*} P<0.05$ vs Pre, ${ }^{\dagger} P<0.05$ vs teneligliptin

diastolic blood pressures were significantly decreased in T/C compared with Pre. There were no changes in pulse rate or amount of water intake among all groups. Table 4 shows the changes in blood and urine test results. Creatinine was significantly increased in $\mathrm{T}$ and $\mathrm{T} / \mathrm{C}$ compared with Pre, and eGFR was significantly decreased. Uric acid was significantly decreased in T/C compared with Pre and T. AST, LDH and ALP showed no changes, whereas ALT was significantly decreased in T/C compared with Pre and T. LDL-C and triglycerides showed significant changes, with a tendency to decrease with treatment, whereas HDL-C was significantly decreased in $\mathrm{T}$ and $\mathrm{T} / \mathrm{C}$ compared with Pre. Urine protein was significantly decreased in $\mathrm{T} / \mathrm{C}$ compared with Pre, whereas urine albumin did not change. Urine glucose was significantly increased in T/C compared with Pre and T. Acetoacetic acid was significantly decreased in $\mathrm{T}$ compared with Pre, and that of T/C was significantly increased compared with T. 3-Hydroxybutyric acid and total ketone bodies were significantly increased in T/C compared with $\mathrm{T}$. Urinary CPR showed a tendency to decrease with treatment and hs-CRP did not change.

\section{DISCUSSION}

In this study, Japanese patients with type 2 diabetes were treated with the DPP- 4 inhibitor teneligliptin in the short-term, followed by concurrent treatment with the SGLT2 inhibitor canagliflozin. As the results, subjects showed improvement in blood glucose level, a decrease in required insulin dose along with an improvement in early phase insulin secretion, promotion of GLP-1 secretion and suppression of excessive glucagon secretion. In addition, decreases in body weight, blood pressure and uric acid were found.

To date, no studies have examined plasma glucagon levels during treatment with canagliflozin using an appropriate measurement method. In this study, before drug administration, the plasma glucagon increased until 90 min after a meal, while this excessive secretion was significantly reduced after teneligliptin treatment and the add-on therapy of canagliflozin. Several previous studies reported that treatment with an SGLT2 inhibitor (dapagliflozin, luseogliflozin or empagliflozin) for 1-12 weeks significantly increased fasting or postprandial plasma glucagon levels in type 2 diabetes patients [8-14]. Two studies reported that canagliflozin treatment also increased plasma glucagon in Japanese and American diabetic patients $[15,16]$. By contrast, one study using ipragliflozin reported no changes in fasting plasma glucagon in Japanese type 2 diabetes patients [17]. In addition, plasma glucagon levels during MTT did not change after 24-week addition of canagliflozin to teneligliptin [18]. In 
Table 4 Changes in the parameters

\begin{tabular}{lcccc}
\hline & Pre & Teneligliptin & Teneli/Cana & $P$ value \\
\hline BUN $(\mathrm{mg} / \mathrm{dL})$ & $13.1 \pm 2.4$ & $12.6 \pm 3.8$ & $14.2 \pm 4.4$ & 0.18 \\
Creatinine $(\mathrm{mg} / \mathrm{dL})$ & $0.64 \pm 0.16$ & $0.71 \pm 0.22^{*}$ & $0.74 \pm 0.22^{*}$ & 0.0007 \\
eGFR $\left(\mathrm{mL} / \mathrm{min} / \mathrm{l} .73 \mathrm{~m}^{2}\right)$ & $78.1 \pm 14.6$ & $72.6 \pm 17.3^{*}$ & $68.9 \pm 17.1^{*} \dagger$ & 0.0001 \\
Uric acid $(\mathrm{mg} / \mathrm{dL})$ & $5.9 \pm 1.0$ & $6.1 \pm 1.4$ & $4.7 \pm 1.2^{*} \dagger$ & 0.0003 \\
AST $(\mathrm{IU} / \mathrm{L})$ & $35.3 \pm 20.1$ & $32.1 \pm 14.5$ & $31.8 \pm 16.4$ & 0.21 \\
ALT $(\mathrm{IU} / \mathrm{L})$ & $40.1 \pm 32.7$ & $36.7 \pm 29.5$ & $33.5 \pm 28.0^{*} \dagger$ & 0.020 \\
$\gamma$-GTP $(\mathrm{IU} / \mathrm{L})$ & $58.3 \pm 55.4$ & $50.2 \pm 46.6$ & $44.9 \pm 37.9$ & 0.037 \\
LDH & $212.1 \pm 50.1$ & $206.1 \pm 44.3$ & $194.2 \pm 54.9$ & 0.099 \\
ALP & $235.9 \pm 66.6$ & $234.9 \pm 65.1$ & $225.0 \pm 62.0$ & 0.25 \\
LDL-C $(\mathrm{mg} / \mathrm{dL})$ & $137.8 \pm 21.4$ & $129.5 \pm 19.6$ & $128.5 \pm 18.4$ & 0.018 \\
HDL-C $(\mathrm{mg} / \mathrm{dL})$ & $51.6 \pm 8.1$ & $47.4 \pm 9.8^{*}$ & $46.7 \pm 10.0^{*}$ & 0.006 \\
Triglyceride $(\mathrm{mg} / \mathrm{dL})$ & $105.5 \pm 26.4$ & $90.3 \pm 17.4$ & $88.4 \pm 18.3$ & 0.028 \\
Urine protein $(\mathrm{mg} /$ day) & $57.0 \pm 40.0$ & $47.0 \pm 26.3$ & $41.0 \pm 26.5^{*}$ & 0.021 \\
Urine albumin $(\mathrm{mg} / \mathrm{day})$ & $31.9 \pm 53.6$ & $14.3 \pm 19.6$ & $16.8 \pm 32.1$ & 0.084 \\
Urine glucose $(\mathrm{g} / \mathrm{day})$ & $0.9 \pm 1.9$ & $0.4 \pm 0.6$ & $40.0 \pm 20.3^{*} \dagger$ & $<0.0001$ \\
Acetoacetic acid $(\mu \mathrm{mol} / \mathrm{L})$ & $98.7 \pm 43.3$ & $66.9 \pm 33.5^{*}$ & $112.3 \pm 47.9 \dagger$ & 0.006 \\
3-Hydroxybutyric acid $(\mu \mathrm{mol} / \mathrm{L})$ & $273.4 \pm 155.4$ & $218.3 \pm 153.8$ & $393.5 \pm 195.3 \dagger$ & 0.005 \\
Total ketone body $(\mu \mathrm{mol} / \mathrm{L})$ & $372.1 \pm 197.1$ & $285.2 \pm 185.9$ & $505.8 \pm 241.4 \dagger$ & 0.005 \\
hs-CRP $(\mathrm{ng} / \mathrm{mL})$ & $2574 \pm 2724$ & $2144 \pm 2932$ & $1849 \pm 2380$ & 0.21 \\
U-CPR $(\mu \mathrm{g} / \mathrm{day})$ & $57.0 \pm 40.0$ & $47.0 \pm 26.3$ & $41.0 \pm 26.5$ & 0.021 \\
\hline Val & & & &
\end{tabular}

Values are mean $\pm \mathrm{SD}$

Pre premedication, Teneli/Cana combination tablet of teneligliptin and canagliflozin, BUN blood urea nitrogen, $e G F R$ estimated glomerular filtration rate, $A S T$ aspartate transaminase, ALT alanineaminotransferase, $\gamma$-GTP $\gamma$-glutamyltransferase, $L D H$ lactate dehydrogenase, $A L P$ alkaline phosphatase, $L D L-C$ low-density lipoprotein cholesterol, $H D L-C$ highdensity lipoprotein cholesterol, $h s-C R P$ high sensitivity $\mathrm{C}$-reactive protein, $U$-CPR urine C-peptide reactivity ${ }^{*} P<0.05$ vs Pre, ${ }^{\dagger} P<0.05$ vs teneligliptin

the above studies [8-18], glucagon was measured by radioimmunoassay (RIA), or the measurement method was not described. Because RIA for glucagon determination shows crossreactivity against several proglucagon-derived fragments, a large difference in glucagon results was noted in the same sample between RIA and sandwich ELISA $[19,20]$. Sandwich ELISA is clearly reported to more accurate measurement system for glucagon assay than conventional
RIA by comparing the most accurate liquid chromatography-high- resolution mass spectrometry assay [20]. Thus, the incongruent results between our study and the previous studies are considered to be attributable to the difference in measurement methods used. In the present study, we employed the sandwich ELISA as the most reliable method, and plasma processing to avoid measurement interference by the presence of mixed substances in the 
plasma was appropriately performed. Teneligliptin suppressed glucagon secretion, as expected, and concurrent use of canagliflozin did not enhance glucagon secretion. Among SGLT2 inhibitors, canagliflozin has the highest inhibitory activity against SGLT1 [21]. SGLT1 but not SGLT2 is expressed in the murine $\alpha \mathrm{TC} 1$ cell line and human and murine pancreatic islets [22, 23]. Canagliflozin, the dual SGLT1/ SGLT2 inhibitor sotagliflozin and small interfering RNA for SGLT1 reduced glucagon secretion from aTC1 cells [22]. A single administration of sotagliflozin to type 2 diabetes patients did not change plasma glucagon [24]. We consider that the suppression of glucagon secretion by concurrent use of teneligliptin and canagliflozin also contributed to reductions in the required insulin dose and insulin resistance, leading to an improvement in blood glucose. Thus far, many studies have reported that SGLT2 inhibitors increased plasma glucagon; however, further studies are required to determine whether this is due to differences in measurement methods and whether there is a subclass effect among SGLT2 inhibitors.

In the present study, plasma active GLP-1 levels in MTTs were increased after treatment with teneligliptin, and were further significantly increased with concurrent use of canagliflozin. In particular, plasma active GLP-1 was high at 90-120 min after a meal. In a previous study in Japanese diabetic patients receiving teneligliptin and canagliflozin in the reverse order of administration to our study, the plasma active GLP-1 level during MTT increased 3 days after canagliflozin treatment, and it was further increased after the addition of teneligliptin [25]. The mechanism of canagliflozin's effect to increase plasma GLP-1 levels is postulated as follows. SGLT1 is abundantly expressed in the small intestine and absorbs glucose from the lumen [26]. Canagliflozin has a certain inhibitory activity against SGLT1 as described above, and thus the inhibition of SGLT1 in the intestine may lead to increased glucose absorption in the lower intestinal tract of humans, resulting in stimulation of L-cells abundant further along the lower intestine, which increases GLP-1 secretion. In DPP-4-deficient F344 rats, the plasma active GLP-1 level after oral glucoseloading was significantly increased after canagliflozin administration compared with baseline [27]. It was also reported that the residual amount of glucose in the small intestine $1 \mathrm{~h}$ after sucrose-loading was significantly increased in Sprague-Dawley rats administered canagliflozin, but not tofogliflozin [27]. Sotagliflozin also increased plasma active GLP-1 in type 2 diabetes patients [24, 28]. Although further GLP-1 secretion in T/C compared to $\mathrm{T}$ is desirable for blood glucose control, further studies including a cross-over design using highly selective SGLT2 inhibitor like empagliflozin are necessary to determine whether there is a subclass effect among SGLT2 inhibitors.

The indices of insulin secretion ability $(\Delta \mathrm{CPR}-30 \mathrm{~min} / \Delta$ glucose-30 $\mathrm{min}$ and SUIT) were significantly greater in $\mathrm{T}$ and $\mathrm{T} / \mathrm{C}$ compared with baseline. Another index of insulin secretion (HOMA2-\%B) was significantly increased in $\mathrm{T}$ compared with baseline, and that of $\mathrm{T} / \mathrm{C}$ was significantly higher than that of $\mathrm{T}$ monotherapy. On the other hand, an index of insulin resistance (CPR-IR) was significantly increased (insulin resistance improved) by combination therapy with $\mathrm{T} / \mathrm{C}$ compared with $\mathrm{T}$. In this study, plasma glucose significantly improved in $\mathrm{T}$, and the total amount of insulin secretion did not significantly change; however, the total amount of insulin secretion in response to glucose (AUC-CPR $\mathrm{C}_{0-180} /$ AUC-glucose $_{0-180}$ ) significantly increased. We suggest that the concurrent use of teneligliptin and canagliflozin led to further improvement in blood glucose levels and reductions in the required insulin dose and insulin resistance, possibly leading to the prevention of future exhaustion of pancreatic $\beta$-cells. The proposed mechanisms of improvement in insulin resistance by SGLT2 inhibitors include the following: improvement of fatty liver $[29,30]$, reduction in the amount of fat including visceral fat [31] and reduction in the amount of triglycerides in the muscle [32]. Reduction of serum ALT and body weight in this study may explain, at least in part, the improvement of insulin resistance.

In this study, the decrease in serum uric acid, increase in ketone bodies and no changes of plasma ghrelin and des-acyl ghrelin were 
observed. Serum uric acid was reported to decrease significantly, as observed in this study, following SGLT2 inhibitor administration, attributable to the increase in the urinary excretion rate of uric acid because of the inhibition of uric acid reabsorption [33]. Although increases in serum ketone bodies following SGLT2 inhibitor administration, as in this study, have often been reported [34-36]. the risk of diabetic ketoacidosis did not increase in a meta-analysis of randomized controlled trials and was equal to that observed for DPP-4 inhibitors [35, 37]. There are only two reports, including this study, which examined plasma ghrelin levels before and after administration of a SGLT2 inhibitor. The results of the other report, which we reported previously using ipragliflozin, are similar to the present study [38].

The limitations of this study include its small sample size, uncontrolled observational study design, insufficient observation period, and assessment under strict dietary therapy during hospitalization. Further evaluation of concurrent use of DPP-4 and SGLT2 inhibitors will likely be of interest.

\section{CONCLUSIONS}

We suggest that combination therapy with a DPP-4 inhibitor and an SGLT2 inhibitor, particularly canagliflozin, which has specific inhibitory activity against SGLT1, has beneficial effects on plasma glucagon and GLP-1 levels, thereby improving blood glucose control in type 2 diabetes patients.

\section{ACKNOWLEDGEMENTS}

We thank the participants of the study.

Funding. This study along with the Rapid Service Fee was funded by Mitsubishi Tanabe Pharma Corporation and a Grant for Clinical Research from Miyazaki University Hospital. All authors had full access to all of the data in this study and take complete responsibility for the integrity of the data and accuracy of the data analysis.

Authorship. All named authors meet the International Committee of Medical Journal Editors (ICMJE) criteria for authorship for this article, take responsibility for the integrity of the work as a whole, and have given their approval for this version to be published.

Disclosures. Masamitsu Nakazato has received research support from Mitsubishi Tanabe Pharma Corporation. Hiroaki Ueno has received research support from Miyazaki University Hospital. Tomoho Noda, Emi Ebihara, Keisuke Sadohara, Yuri Tanaka, Yuuma Nagatomo, Yousuke Murakami, Shinichi Yonamine, Wakaba Tsuchimochi, Hideyuki Sakoda, and Hideki Yamaguchi have nothing to disclose.

Compliance with Ethics Guidelines. This study was approved by the Ethics Review Committee of the University of Miyazaki and is registered in the University Hospital Medical Information Network Clinical Trials Registry as UMIN000030043. All procedures were in accordance with the ethical standards of the responsible committee on human experimentation (institutional and national) and the Helsinki Declaration of 1964, as revised in 2013. Written informed consent to participate was obtained from all patients.

Data Availability. The datasets obtained and/or analysed during the current study are available from the corresponding author on reasonable request.

Open Access. This article is distributed under the terms of the Creative Commons Attribution-NonCommercial 4.0 International License (http://creativecommons.org/licenses/ by-nc/4.0/), which permits any noncommercial use, distribution, and reproduction in any medium, provided you give appropriate credit to the original author(s) and the source, provide a link to the Creative Commons license, and indicate if changes were made. 


\section{REFERENCES}

1. Seino $Y$, Kuwata H, Yabe D. Incretin-based drugs for type 2 diabetes: focus on East Asian perspectives. J Diabetes Investig. 2016;7:102-9.

2. Wilding J, Fernando K, Milne N, et al. SGLT2 inhibitors in type 2 diabetes management: key evidence and implications for clinical practice. Diabetes Ther. 2018;9:1757-73.

3. Neal B, Perkovic V, Mahaffey KW, et al. Canagliflozin and cardiovascular and renal events in type 2 diabetes. N Engl J Med. 2017;377:644-57.

4. Tsuchimochi W, Ueno H, Yamashita E, et al. Teneligliptin improves glycemic control with the reduction of postprandial insulin requirement in Japanese diabetic patients. Endocr J. 2015;62:13-20.

5. Yamada Y, Fukuda K, Fujimoto S, et al. SUIT, secretory units of islets in transplantation: an index for therapeutic management of islet transplanted patients and its application to type 2 diabetes. Diabetes Res Clin Pract. 2006;74:222-6.

6. Okura T, Nakamura R, Fujioka Y, et al. CPR-IR is an insulin resistance index that is minimally affected by hepatic insulin clearance-a preliminary research. PLoS One. 2018;13:e0197663.

7. Wallace TM, Levy JC, Matthews DR. Use and abuse of HOMA modeling. Diabetes Care. 2004;27:1487-95.

8. Ferrannini E, Muscelli E, Frascerra S, et al. Metabolic response to sodium-glucose cotransporter 2 inhibition in type 2 diabetic patients. J Clin Invest. 2014;124:499-508.

9. Nishimura R, Omiya H, Sugio K, Ubukata M, Sakai S, Samukawa Y. Sodium-glucose cotransporter 2 inhibitor luseogliflozin improves glycaemic control, assessed by continuous glucose monitoring, even on a low-carbohydrate diet. Diabetes Obes Metab. 2016;18:702-6.

10. Nishimura R, Osonoi T, Kanada S, et al. Effects of luseogliflozin, a sodium-glucose co-transporter 2 inhibitor, on 24-h glucose variability assessed by continuous glucose monitoring in Japanese patients with type 2 diabetes mellitus: a randomized, double-blind, placebo-controlled, crossover study. Diabetes Obes Metab. 2015;17:800-4.

11. Al Jobori H, Daniele G, Adams J, et al. Determinants of the increase in ketone concentration during SGLT2 inhibition in NGT, IFG and T2DM patients. Diabetes Obes Metab. 2017;19:809-13.
12. Alsalim W, Persson M, Ahren B. Different glucagon effects during DPP-4 inhibition versus SGLT-2 inhibition in metformin-treated type 2 diabetes patients. Diabetes Obes Metab. 2018;20:1652-8.

13. Forst T, Falk A, Andersen G, et al. Effects on alphaand beta-cell function of sequentially adding empagliflozin and linagliptin to therapy in people with type 2 diabetes previously receiving metformin: an exploratory mechanistic study. Diabetes Obes Metab. 2017;19:489-95.

14. Hansen L, Iqbal N, Ekholm E, Cook W, Hirshberg B. Postprandial dynamics of plasma glucose, insulin, and glucagon in patients with type 2 diabetes treated with saxagliptin plus dapagliflozin add-on to metformin therapy. Endocr Pract. 2014;20:1187-97.

15. Martinez R, Al-Jobori H, Ali AM, et al. Endogenous glucose production and hormonal changes in response to canagliflozin and liraglutide combination therapy. Diabetes. 2018;67:1182-9.

16. Okahata S, Sakamoto K, Mitsumatsu T, Kondo Y, Tanaka S, Shiba T. Mechanistic insights from sequential combination therapy with a sodium glucose co-transporter- 2 inhibitor and a dipeptidyl peptidase-4 inhibitor: results from the CANARIS Trial using canagliflozin and teneligliptin. Diabetes Obes Metab. 2019;21:388-92.

17. Ishihara H, Yamaguchi S, Nakao I, Okitsu A, Asahina S. Efficacy and safety of ipragliflozin as add-on therapy to insulin in Japanese patients with type 2 diabetes mellitus (IOLITE): a multi-centre, randomized, placebo-controlled, double-blind study. Diabetes Obes Metab. 2016;18:1207-16.

18. Kadowaki T, Inagaki N, Kondo K, et al. Efficacy and safety of canagliflozin as add-on therapy to teneligliptin in Japanese patients with type 2 diabetes mellitus: results of a 24-week, randomized, doubleblind, placebo-controlled trial. Diabetes Obes Metab. 2017;19:874-82.

19. Matsuo T, Miyagawa J, Kusunoki Y, et al. Postabsorptive hyperglucagonemia in patients with type 2 diabetes mellitus analyzed with a novel enzymelinked immunosorbent assay. J Diabetes Investig. 2016;7:324-31.

20. Miyachi A, Kobayashi M, Mieno E, et al. Accurate analytical method for human plasma glucagon levels using liquid chromatography-high resolution mass spectrometry: comparison with commercially available immunoassays. Anal Bioanal Chem. 2017;409:5911-8.

21. Grempler R, Thomas L, Eckhardt M, et al. Empagliflozin, a novel selective sodium glucose cotransporter-2 (SGLT-2) inhibitor: characterisation and 
comparison with other SGLT-2 inhibitors. Diabetes Obes Metab. 2012;14:83-90.

22. Suga T, Kikuchi O, Kobayashi M, et al. SGLT1 in pancreatic alpha cells regulates glucagon secretion in mice, possibly explaining the distinct effects of SGLT2 inhibitors on plasma glucagon levels. Mol Metab. 2019;19:1-12.

23. Kuhre RE, Ghiasi SM, Adriaenssens AE, et al. No direct effect of SGLT2 activity on glucagon secretion. Diabetologia. 2019;62:1011-23.

24. Zambrowicz B, Freiman J, Brown PM, et al. LX4211, a dual SGLT1/SGLT2 inhibitor, improved glycemic control in patients with type 2 diabetes in a randomized, placebo-controlled trial. Clin Pharmacol Ther. 2012;92:158-69.

25. Takebayashi K, Hara K, Terasawa T, et al. Effect of canagliflozin on circulating active GLP-1 levels in patients with type 2 diabetes: a randomized trial. Endocr J. 2017;64:923-31.

26. Chen J, Williams S, Ho S, et al. Quantitative PCR tissue expression profiling of the human SGLT2 gene and related family members. Diabetes Ther. 2010;1:57-92.

27. Oguma T, Kuriyama C, Nakayama K, et al. Changes in glucose-induced plasma active glucagon-like peptide-1 levels by co-administration of sodiumglucose cotransporter inhibitors with dipeptidyl peptidase- 4 inhibitors in rodents. J Pharmacol Sci. 2016;132:255-61.

28. Zambrowicz B, Lapuerta P, Strumph P, et al. LX4211 therapy reduces postprandial glucose levels in patients with type 2 diabetes mellitus and renal impairment despite low urinary glucose excretion. Clin Ther. 2015;37(71-82):e12.

29. Shibuya T, Fushimi N, Kawai M, et al. Luseogliflozin improves liver fat deposition compared to metformin in type 2 diabetes patients with non-alcoholic fatty liver disease: a prospective randomized controlled pilot study. Diabetes Obes Metab. 2018;20:438-42.

30. Lee PCH, Gu Y, Yeung MY, et al. Dapagliflozin and empagliflozin ameliorate hepatic dysfunction among chinese subjects with diabetes in part through glycemic improvement: a single-center, retrospective, observational study. Diabetes Ther. 2018;9:285-95.

31. Koike Y, Shirabe SI, Maeda H, et al. Effect of canagliflozin on the overall clinical state including insulin resistance in Japanese patients with type 2 diabetes mellitus. Diabetes Res Clin Pract. 2019;149:140-6.

32. O'Brien TP, Jenkins EC, Estes SK, et al. Correcting postprandial hyperglycemia in Zucker diabetic fatty rats with an SGLT2 inhibitor restores glucose effectiveness in the liver and reduces insulin resistance in skeletal muscle. Diabetes. 2017;66:1172-84.

33. Zhao Y, Xu L, Tian D, et al. Effects of sodium-glucose co-transporter 2 (SGLT2) inhibitors on serum uric acid level: a meta-analysis of randomized controlled trials. Diabetes Obes Metab. 2018;20:458-62.

34. Kawamori R, Haneda M, Suzaki K, et al. Empagliflozin as add-on to linagliptin in a fixed-dose combination in Japanese patients with type 2 diabetes: glycaemic efficacy and safety profile in a 52-week, randomized, placebo-controlled trial. Diabetes Obes Metab. 2018;20:2200-9.

35. Polidori D, Iijima $\mathrm{H}$, Goda $\mathrm{M}$, Maruyama N, Inagaki $\mathrm{N}$, Crawford PA. Intra- and inter-subject variability for increases in serum ketone bodies in patients with type 2 diabetes treated with the sodium glucose co-transporter 2 inhibitor canagliflozin. Diabetes Obes Metab. 2018;20:1321-6.

36. Prattichizzo F, De Nigris V, Micheloni S, La Sala L, Ceriello A. Increases in circulating levels of ketone bodies and cardiovascular protection with SGLT2 inhibitors: is low-grade inflammation the neglected component? Diabetes Obes Metab. 2018;20:2515-22.

37. Kim YG, Jeon JY, Han SJ, Kim DJ, Lee KW, Kim HJ. Sodium-glucose co-transporter-2 inhibitors and the risk of ketoacidosis in patients with type 2 diabetes mellitus: a nationwide population-based cohort study. Diabetes Obes Metab. 2018;20:1852-8.

38. Ueno $H$, Nakazato $H$, Ebihara E, et al. Effects of ipragliflozin on postprandial glucose metabolism and gut peptides in type 2 diabetes: a pilot study. Diabetes Ther. 2018;9:403-11. 